\title{
A Local Estimate for the Maximal Function in Lebesgue Spaces with EXP-Type Exponents
}

\begin{abstract}
Alberto Fiorenza ${ }^{1,2}$
${ }^{1}$ Dipartimento di Architettura, Università di Napoli, Via Monteoliveto 3, 80134 Napoli, Italy

${ }^{2}$ Istituto per le Applicazioni del Calcolo "Mauro Picone", Sezione di Napoli, Consiglio Nazionale delle Ricerche,
\end{abstract} Via Pietro Castellino 111, 80131 Napoli, Italy

Correspondence should be addressed to Alberto Fiorenza; fiorenza@unina.it

Received 18 March 2015; Accepted 3 May 2015

Academic Editor: Henryk Hudzik

Copyright (c) 2015 Alberto Fiorenza. This is an open access article distributed under the Creative Commons Attribution License, which permits unrestricted use, distribution, and reproduction in any medium, provided the original work is properly cited.

It is proven that if $1 \leq p(\cdot)<\infty$ in a bounded domain $\Omega \subset \mathbb{R}^{n}$ and if $p(\cdot) \in \operatorname{EXP}_{a}(\Omega)$ for some $a>0$, then given $f \in L^{p(\cdot)}(\Omega)$, the Hardy-Littlewood maximal function of $f, M f$, is such that $p(\cdot) \log (M f) \in \operatorname{EXP}_{a /(a+1)}(\Omega)$. Because $a /(a+1)<1$, the thesis is slightly weaker than $(M f)^{\lambda p(\cdot)} \in L^{1}(\Omega)$ for some $\lambda>0$. The assumption that $p(\cdot) \in \operatorname{EXP}_{a}(\Omega)$ for some $a>0$ is proven to be optimal in the framework of the Orlicz spaces to obtain $p(\cdot) \log (M f)$ in the same class of spaces.

\section{Introduction}

Let $\Omega \subset \mathbb{R}^{n}$ be a bounded domain. By $p(\cdot)$, we denote a finite exponent function on $\Omega$, that is, a Lebesgue measurable function $p(\cdot): \Omega \rightarrow\left[1, \infty\left[\right.\right.$. The space $L^{p(\cdot)}(\Omega)$ is defined as the set of Lebesgue measurable functions $f$ on $\Omega$ such that

$$
\int_{\Omega}|\mu f(x)|^{p(x)} d x<\infty
$$

for some $\mu>0$.

The Hardy-Littlewood maximal operator of a function $f \in L_{\text {loc }}^{1}\left(\mathbb{R}^{n}\right)$ is defined by

$$
M f(x)=\sup _{\mathrm{Q} \ni x} f_{Q}|f(y)| d y, \quad x \in \mathbb{R}^{n},
$$

where the supremum is taken over all cubes $Q \subset \mathbb{R}^{n}$ that contain $x$ and whose sides are parallel to the coordinate axes (the symbol $f_{Q}$ denotes $1 /|Q|$, and for a Lebesgue measurable $E \subset \mathbb{R}^{n}$, the symbol $|E|$ denotes its Lebesgue measure). If $f \in L^{1}(\Omega)$ (and, in particular, also if $f \in L^{p(\cdot)}(\Omega)$; see [1, Proposition 2.41 page 36]), then by $M f$, we mean the maximal operator computed on the extension of $f$ by zero outside $\Omega$; in this case, it is known (see, e.g., [2, 8.15 page 43] or [3]) that

$$
\begin{gathered}
(M f)^{q} \in L^{1}(E) \\
\forall 0<q<1, \quad \forall E \subset \mathbb{R}^{n}, \quad|E|<\infty, \\
\int_{E} M f(x)^{q} d x \leq c_{q}|E|^{1-q}\left(\int_{\Omega}|f(x)| d x\right)^{q} .
\end{gathered}
$$

The main classical result regarding the maximal operator is that it is bounded in every Lebesgue space with (constant) exponent greater that 1 (see, e.g., [2, 4-7] and references therein for the main results regarding the maximal operator). The extension of this result in the framework of variable Lebesgue spaces theory has been intensively studied (see, e.g., [1, Chapter 3] or [8, Chapter 4], [9, Chapter 1] for a collection of results regarding this topic; see also the survey [10]).

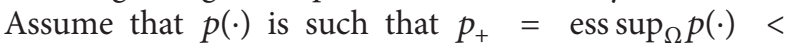
$\infty$. It is well-known that $M$ is not necessarily bounded in $L^{p(\cdot)}(\Omega)$ even if, in addition, $p_{-}=\operatorname{essinf}_{\Omega} p(\cdot)>1$ and $p(\cdot)$ is continuous (see [11]; the same example is also presented in [1, Example 4.43 page 160]). A well-known sufficient condition for boundedness of the maximal operator for exponents $p(\cdot)$ such that $p_{-}>1$ is expressed in terms of the so-called log-Hölder continuity, which may be required to 
the function $1 / p(\cdot)$ (see [12] and references therein for details). However, sufficient conditions that do not require continuity do exist (see, e.g., $[13,14])$. In [15], another class of exponents that ensures the boundedness of the maximal operator for bounded domains has been presented.

However, we can assert that

$$
\begin{aligned}
& p_{+}<\infty, \\
& f \in L^{p(\cdot)}(\Omega) \Longrightarrow \exists \lambda>0:(M f)^{\lambda p(\cdot)} \in L^{1}(\Omega) .
\end{aligned}
$$

In fact, by (3), for $0<q<1$, we have

$$
\begin{aligned}
f & \in L^{p(\cdot)}(\Omega) \Longrightarrow f \in L^{1}(\Omega) \Longrightarrow(M f)^{q} \\
& =(M f)^{p_{+} q / p_{+}} \in L^{1}(\Omega) \Longrightarrow(M f)^{p(\cdot) q / p_{+}} \in L^{1}(\Omega) .
\end{aligned}
$$

Note that from (5), one gets that, for $0<\beta<1$, if $p_{+}<\infty$,

$$
\begin{aligned}
& f \in L^{p(\cdot)}(\Omega) \Longrightarrow \exists \lambda>0: \exp \left((\lambda p(\cdot)|\log (M f)|)^{\beta}\right) \\
& \quad \in L^{1}(\Omega) .
\end{aligned}
$$

For unbounded (and possibly discontinuous) exponents, (7) is not generically true (see the example presented below). In this note, we prove that, for some $0<\beta<1$, (7) holds true under an assumption of exponential integrability of the exponent. We observe that some type of high integrability of the exponent is needed; in fact, the result is not true if it is only assumed that $p(\cdot)$ is contained in a Lebesgue space with any finite, constant exponent. For instance, if $p(x)=x^{-b}+1$ on $(0,1), b>0$, then $f(x)=(1 / \sqrt{x})^{1 /\left(x^{-b}+1\right)}, x \in(0,1)$, is such that $f \in L^{p(\cdot)}(0,1)$ but for every $\lambda>0$, it is

$$
1<\left(\int_{0}^{1} f(x) d x\right)^{\lambda}<\infty
$$

Therefore, for any $0<\beta<1$,

$$
\begin{aligned}
& \int_{0}^{1} \exp \left((\lambda p(x)|\log (M f(x))|)^{\beta}\right) d x \\
& \quad \geq \int_{0}^{1} \exp \left(\left(\lambda\left(x^{-b}+1\right) \log \left(\int_{0}^{1} f\right)\right)^{\beta}\right) d x \\
& \quad \geq c_{b, \beta} \int_{0}^{1}\left[\left(\lambda\left(x^{-b}+1\right) \log \left(\int_{0}^{1} f\right)\right)^{\beta}\right]^{1 / b \beta} d x \\
& \quad=c_{b, \beta} \int_{0}^{1} \lambda^{1 / b}\left(x^{-b}+1\right)^{1 / b} \log ^{1 / b}\left(\int_{0}^{1} f\right) d x=\infty
\end{aligned}
$$

For other results regarding the maximal operator that are specific to unbounded exponents, see [16].

\section{The Local Estimate}

In the following, three prerequisites are necessary. The first is the well-known Fefferman-Stein inequality ([17]; see also [18])

$$
\begin{array}{r}
\int_{\mathbb{R}^{n}} M f(x)^{q}|g(x)| d x \leq c_{q} \int_{\mathbb{R}^{n}}|f(x)|^{q} M g(x) d x, \\
f, g \in L_{\mathrm{loc}}^{1}\left(\mathbb{R}^{n}\right),
\end{array}
$$

which is true for $q>1$ (here $q$ is constant). The second is a well-known extrapolation characterisation (see, e.g., [19-23]) of $\operatorname{EXP}_{a}(\Omega), a>0$, the Orlicz space of the functions, which can be characterised in one of the two following equivalent ways:

$$
\begin{aligned}
\limsup _{k \rightarrow \infty} \frac{1}{k^{1 / a}}\left(f_{\Omega}|h(x)|^{k} d x\right)^{1 / k} \\
<\infty \Longleftrightarrow \exists \mu>0: f_{\Omega} \exp \left(|\mu h(x)|^{a}\right) d x<\infty .
\end{aligned}
$$

We remark that the growth of integrability of the functions in $\operatorname{EXP}_{a}(\Omega), 0<a<1$, is somehow connected with the $A_{\infty}$ property for Young functions; see [24]. Finally, the third tool is an elementary inequality. For $k \geq 1, \alpha \geq 1$, and a positive constant

$$
\gamma_{k, \alpha}=k^{\alpha(k-1)+1} \exp \left(-k^{\alpha}\right),
$$

(as usual, subscripts indicate the dependence of the constant on the variables involved, which may change from line to line), it is

$$
\log ^{k} \xi \leq k^{\alpha k}+\gamma_{k, \alpha}\left(\xi-\exp \left(k^{\alpha}\right)\right) \quad \forall \xi \geq \exp \left(k^{\alpha}\right) .
$$

This inequality is a direct consequence of the concavity of the $k$ th power of the logarithm in $(\exp (k-1), \infty)$.

We are now ready for the proof of the following theorem.

Theorem 1. Let $\Omega$ be a bounded domain in $\mathbb{R}^{n}$, and let $p(\cdot) \in$ $\operatorname{EXP}_{a}(\Omega)$ for some $a>0$ be such that $1 \leq p(\cdot)<\infty$. If $f \in$ $L^{p(\cdot)}(\Omega), f \neq \equiv 0$, then $p(\cdot) \log (M f) \in \operatorname{EXP}_{a /(a+1)}(\Omega)$.

Proof. Let us first assume that $p_{-}>1$. Considering that $p(\cdot) \in$ $\operatorname{EXP}_{a}(\Omega)$, for every $k \geq\left|\log \|f\|_{L^{1}(\Omega)}\right|$ we have

$$
\begin{aligned}
& \left(\frac{1}{|\Omega|} \int_{\{x \in \Omega: M f(x) \leq \exp (k)\}} p(x)^{k}|\log M f(x)|^{k} d x\right)^{1 / k} \\
& \quad \leq\left(\frac{1}{|\Omega|}\right. \\
& \left.\cdot \int_{\{x \in \Omega: M f(x) \leq \exp (k)\}} p(x)^{k} \log ^{k}(\exp (k)) d x\right)^{1 / k} \\
& \quad=\left(\frac{1}{|\Omega|} \int_{\{x \in \Omega: M f(x) \leq \exp (k)\}} p(x)^{k} k^{k} d x\right)^{1 / k} \\
& \quad \leq k\left(f_{\Omega} p(x)^{k} d x\right)^{1 / k} \leq k c_{p(\cdot)} k^{1 / a}=c_{p(\cdot)} k^{1+1 / a} .
\end{aligned}
$$


On the other hand, for any $q>1$ and $r>1$ such that $q r<p_{-}$, the following inequalities hold (we are going to apply (13) with the choices $\alpha=1$ and $\xi=M f(x)^{q}$; note that because we work on the set in which $M f(x)>\exp (k)$, it is also true that $\left.M f(x)^{q}>\exp (k)\right)$ :

$$
\begin{aligned}
& \left(\frac{1}{|\Omega|} \int_{\{x \in \Omega: M f(x)>\exp (k)\}} p(x)^{k}|\log M f(x)|^{k} d x\right)^{1 / k} \\
& =\frac{1}{q}\left(\frac{1}{|\Omega|} \int_{\{x \in \Omega: M f(x)>\exp (k)\}} p(x)^{k}\right. \\
& \left.\cdot \log ^{k} M f(x)^{q} d x\right)^{1 / k} \leq \frac{1}{q}\left(\frac{1}{|\Omega|}\right. \\
& \cdot \int_{\{x \in \Omega: M f(x)>\exp (k)\}} p(x)^{k} \\
& \left.\cdot\left[k^{k}+\gamma_{k, 1}\left(M f(x)^{q}-\exp (k)\right)\right] d x\right)^{1 / k}=\frac{1}{q}\left(\frac{1}{|\Omega|}\right. \\
& \cdot \int_{\{x \in \Omega: M f(x)>\exp (k)\}} p(x)^{k} k^{k} d x+\frac{1}{|\Omega|} \\
& \cdot \int_{\{x \in \Omega: M f(x)>\exp (k)\}} p(x)^{k} \\
& \left.\cdot \gamma_{k, 1}\left(M f(x)^{q}-\exp (k)\right) d x\right)^{1 / k} \leq \frac{1}{q}\left(\frac{1}{|\Omega|}\right. \\
& \left.\cdot \int_{\{x \in \Omega: M f(x)>\exp (k)\}} p(x)^{k} k^{k} d x\right)^{1 / k}+\frac{1}{q}\left(\frac{1}{|\Omega|}\right. \\
& \cdot \int_{\{x \in \Omega: M f(x)>\exp (k)\}} p(x)^{k} \\
& \left.\cdot \gamma_{k, 1}\left(M f(x)^{q}-\exp (k)\right) d x\right)^{1 / k}
\end{aligned}
$$

and because $\gamma_{k, 1}^{1 / k}=k / e$, by again using the fact that $p(\cdot) \epsilon$ $\operatorname{EXP}_{a}(\Omega)$ and by applying (10),

$$
\begin{aligned}
\leq & \frac{k}{q}\left(f_{\Omega} p(x)^{k} d x\right)^{1 / k}+\frac{k}{e q}\left(\frac{1}{|\Omega|}\right. \\
& \left.\cdot \int_{\{x \in \Omega: M f(x)>\exp (k)\}} p(x)^{k} M f(x)^{q} d x\right)^{1 / k} \leq \frac{k}{q} \\
& \cdot c_{p(\cdot)} k^{1 / a}+\frac{k}{e q}\left(\frac{1}{|\Omega|} \int_{\mathbb{R}^{n}} M f(x)^{q} p(x)^{k}\right. \\
& \left.\cdot \chi_{\{x \in \Omega: M f(x)>\exp (k)\}}(x) d x\right)^{1 / k} \leq \frac{c_{p(\cdot)}}{q} k^{1+1 / a}
\end{aligned}
$$

$$
\begin{aligned}
& +\frac{k}{e q}\left(\frac{c_{q}}{|\Omega|} \int_{\mathbb{R}^{n}}|f(x)|^{q}\right. \\
& \left.\cdot M\left(p(x)^{k} \chi_{\{x \in \Omega: M f(x)>\exp (k)\}}(x)\right) d x\right)^{1 / k} \leq \frac{c_{p(\cdot)}}{q} \\
& \cdot k^{1+1 / a}+\frac{k}{e q}\left(\frac{c_{q}}{|\Omega|} \int_{\Omega}|f(x)|^{q} M\left(p(x)^{k}\right) d x\right)^{1 / k} .
\end{aligned}
$$

By Hölder's inequality and the boundedness of the maximal operator in $L^{r /(r-1)}(\Omega)$,

$$
\begin{aligned}
& \leq \frac{c_{p(\cdot)}}{q} k^{1+1 / a}+\frac{k}{e q} c_{q}^{1 / k}\left(f_{\Omega}|f(x)|^{q r} d x\right)^{1 / k r} \\
& \cdot\left(f_{\Omega} M\left(p(x)^{k}\right)^{r /(r-1)} d x\right)^{(r-1) / k r} \leq \frac{c_{p(\cdot)}}{q} k^{1+1 / a} \\
& +c_{q}^{1 / k} \frac{k}{e q}\left(f_{\Omega}|f(x)|^{q r} d x\right)^{1 / q r \cdot q / k} \\
& \cdot c_{r}\left(f_{\Omega} p(x)^{k r /(r-1)} d x\right)^{(r-1) / k r} \leq \frac{c_{p(\cdot)}}{q} k^{1+1 / a} \\
& +c_{q}^{1 / k} c_{r} \frac{k}{e q}\left(f_{\Omega}|f(x)|^{p_{-}} d x\right)^{1 / p_{-} \cdot q / k} \\
& \cdot c_{p(\cdot)}\left(\frac{k r}{r-1}\right)^{1 / a} \leq \frac{c_{p(\cdot)}}{q} k^{1+1 / a}+c_{a} c_{q}^{1 / k} c_{r} \\
& \quad \cdot \frac{c_{p(\cdot)}}{q}\left(f_{\Omega}|f(x)|^{p_{-}} d x\right)^{1 / p_{-} \cdot q / k} k^{1+1 / a} \leq C k^{1+1 / a},
\end{aligned}
$$

where $C>0$ is a constant that depends on all parameters and $f \in L^{p(\cdot)}(\Omega)$ but is independent of $k \geq 1$.

Combining the estimates obtained above, we obtain

$$
\left(f_{\Omega} p(x)^{k}|\log M f(x)|^{k} d x\right)^{1 / k} \leq C k^{1+1 / a} .
$$

That is, using (11) (with $a$ replaced by $a /(a+1)$ ), we obtain the assertion.

Now, let $p(\cdot) \in \operatorname{EXP}_{a}(\Omega)$ for some $a>0$, and assume $p_{-}=$ 1. If $p_{+}<\infty$, we already proved (see (7)) that the assertion is trivially true. If $p_{+}=\infty$, set

$$
\begin{aligned}
& A=\{x \in \Omega: 1 \leq p(x) \leq 2 \text { a.e. }\}, \\
& B=\{x \in \Omega: p(x)>2 \text { a.e. }\},
\end{aligned}
$$

and observe that $A$ is nonempty (because $p_{-}=1$ ) and $B$ is nonempty (because $p_{+}=\infty$ ). By (3), $(M f)^{2 / 3} \in L^{1}(A)$. Therefore, $(M f)^{p(\cdot) / 3} \in L^{1}(A)$, from which

$$
\exp \left(\left(\left(\frac{p(\cdot)}{3}\right)|\log (M f)|\right)^{a /(a+1)}\right) \in L^{1}(A) .
$$

On the other hand, since $p(\cdot) \in \operatorname{EXP}_{a}(B)$ and ess $\inf _{B} p(\cdot) \geq$ 2 , there exists $\mu>0$ such that

$$
\exp \left((\mu p(\cdot)|\log (M f)|)^{a /(a+1)}\right) \in L^{1}(B) .
$$


In conclusion, since $\Omega=A \cup B, \exp \left((\lambda p(\cdot)|\log (M f)|)^{a /(a+1)}\right) \epsilon$ $L^{1}(\Omega)$ for $\lambda=\min \{1 / 3, \mu\}$.

The heart of Theorem 1 can be stated as follows: if $p(\cdot)$ belongs to some $\operatorname{EXP}_{a}(\Omega)$, then for any $f \in L^{p(\cdot)}(\Omega)$ also $p(\cdot) \log (M f)$ does. From this perspective, our result is optimal: if one assumes that $p(\cdot)$ belongs to any Orlicz space that contains all of the $\operatorname{EXP}_{a}(\Omega)$ 's, it is possible to construct an exponent that is not in any $\operatorname{EXP}_{a}(\Omega)$ and a function $f \in$ $L^{p(\cdot)}(0,1)$ such that $p(\cdot) \log (M f)$ is also not in any $\operatorname{EXP}_{a}(\Omega)$. This statement is the essence of the following result (for the definition of Young functions and the classical embedding theorem for Orlicz spaces; see, e.g., [25]).

Theorem 2. Let $\Phi:[0, \infty[\rightarrow[0, \infty[$ be a Young function such that for all $a \in] 0,1]$

$$
\Phi(t) \leq \exp \left(t^{a}\right) \quad \forall t>t_{a} .
$$

There exists $\left.\left.p(\cdot) \in L^{\Phi}(0,1), p(\cdot) \notin \operatorname{EXP}_{a}(0,1) \forall a \in\right] 0,1\right]$, $p_{-}>1$, and there exists $f \in L^{p^{(\cdot)}}(0,1)$ such that

$$
\left.\left.p(\cdot) \log (M f) \notin \operatorname{EXP}_{a}(0,1) \quad \forall a \in\right] 0,1\right] .
$$

Proof. Set $A(t)=\log (\Phi(\exp (t)))$ for $t$ large. For every $\beta \epsilon$ ] $0,1]$, set $A_{\beta}(t)=\exp (\beta t)$. Then, (22) reads as

$$
A(\log t)-A_{a}(\log t) \leq 0
$$

or, equivalently,

$$
A^{-1}(t) \geq A_{a}^{-1}(t)=\frac{1}{a} \log t
$$

for large $t$. Set

$$
\begin{array}{r}
p(x)=\Phi^{-1}\left(\frac{\Phi(2)}{\sqrt{x}}\right)=\exp \left(A^{-1}\left(\log \left(\frac{\Phi(2)}{\sqrt{x}}\right)\right)\right) \\
x \in(0,1) .
\end{array}
$$

We observe that, trivially, $p(\cdot) \in L^{\Phi}(0,1)$ and $p_{-}=2>1$.

We now prove that $\left.\left.p(\cdot) \notin \operatorname{EXP}_{a}(0,1) \forall a \in\right] 0,1\right]$. Let $a \in$ ]0,1] and $k>0$. Fix $\beta \in] 0, a[$ (for instance, $\beta=a / 2$ can be assumed in the following). For sufficiently small $x$, using (25) with $a$ replaced by $\beta$, we see that

$$
\begin{aligned}
& \exp \left((k p(x))^{a}\right) \\
& =\exp \left(\left(k \exp \left(A^{-1}\left(\log \left(\frac{\Phi(2)}{\sqrt{x}}\right)\right)\right)\right)^{a}\right) \\
& \geq \exp \left(\left(k \exp \left(\frac{1}{\beta} \log \left(\log \left(\frac{\Phi(2)}{\sqrt{x}}\right)\right)\right)\right)^{a}\right) \\
& =\exp \left(\left(k\left(\log \left(\frac{\Phi(2)}{\sqrt{x}}\right)\right)^{1 / \beta}\right)^{a}\right) \\
& \geq \exp \left(\left(2^{1 / a}\left(\log \left(\frac{\Phi(2)}{\sqrt{x}}\right)\right)^{1 / a}\right)^{a}\right)=\frac{\Phi(2)^{2}}{x} .
\end{aligned}
$$

Therefore,

$$
\left.\left.\exp \left((k p(\cdot))^{a}\right) \notin L^{1}(0,1) \quad \forall a \in\right] 0,1\right], \quad \forall k>0 .
$$

Let us now set

$$
f(x)=\left(\frac{1}{\sqrt{x}}\right)^{1 / p(x)} \quad x \in(0,1),
$$

so that $f \in L^{p(\cdot)}(0,1)$. It remains to prove (23). Fix $\lambda>0$, set $k=\lambda \log \left(\int_{0}^{1}|f(x)| d x\right)$, and note that $k>0$. Thus,

$$
\begin{gathered}
\int_{0}^{1} \exp \left((\lambda p(x) \log (M f(x)))^{a}\right) d x \\
\geq \int_{0}^{1} \exp \left((k p(x))^{a}\right) d x=\infty
\end{gathered}
$$

where the last equality results from (28).

\section{Conflict of Interests}

The author declares that there is no conflict of interests regarding the publication of this paper.

\section{Acknowledgment}

On January 27, 2011, Miroslav Krbec, who should have been the second author of this paper, sent me a message that contained an idea for a proof of a local boundedness-type result for the maximal operator in variable exponent Lebesgue spaces. At that time, I was writing a book [1] on variable Lebesgue spaces with David Cruz-Uribe. The collaboration with Mirek had begun several years before, but we never discussed questions related to variable exponents. Unfortunately, I only glanced at that message, and I replied with a short, evasive answer. I thought the idea was nothing special. On March 4, 2011 Mirek again asked me about the idea. On April 14, 2012, Mirek invited me to visit Prague in June, and he asked me once more to consider the idea. At this point, my opinion changed: I decided that the idea was a very good starting point for a project. With this new perspective, I planned to pack my enthusiasm in my luggage and announce to Mirek in June that a new research project concerning variable Lebesgue spaces should be initiated. However, Mirek died on June 17, 2012, one week before my arrival at Prague. For me, his death was a significant loss: I lost both a friend and a collaboration that had given me much joy from both the human and scientific perspectives. I was upset with myself. I had been lazy, and if I had spent time on this idea, we could have published one more paper together. However, my enthusiasm was too late, and the paper went unwritten. After Mirek's death, I spent much effort on another project (again with Mirek as coauthor) that I was working on (the final goal of which was to prove that the small Lebesgue spaces are the correct setting in which to define the optimal dimensional-free gain of integrability for the Sobolev embedding theorem, when the domain is the unit cube in all dimensions). This other project yielded a publication ([26]; for a further development, 
see [27]). The overall result was that, after the original idea about the variable Lebesgue space question, much time passed, and I was again upset with myself. This paper tries to fill the gap left by the significant amount of time that has passed. This work will never make up for my laziness; however, it at last provides a venue for an idea originally proposed by Mirek.

\section{References}

[1] D. V. Cruz-Uribe and A. Fiorenza, Variable Lebesgue Spaces: Foundations and Harmonic Analysis, Birkhäauser, Basel, Switzerland, 2013.

[2] E. M. Stein, Harmonic Analysis: Real-Variable Methods, Orthogonality, and Oscillatory Integrals, vol. 43 of Monographs in Harmonic Analysis, III, Princeton University Press, Princeton, NJ, USA, 1993, with the assistance of T. S. Murphy.

[3] A. Fiorenza and M. Krbec, "On the domain and range of the maximal operator," Nagoya Mathematical Journal, vol. 158, pp. 43-61, 2000.

[4] J. Duoandikoetxea, Fourier Analysis, vol. 29 of Graduate Studies in Mathematics, American Mathematical Society, Providence, RI, USA, 2001, Translated and Revised by: D. Cruz-Uribe from the 1995 Spanish Original.

[5] D. Cruz-Uribe, J. M. Martell, and C. Pérez, Weights, Extrapolation and the Theory of Rubio de Francia, vol. 215 of Operator Theory: Advances and Applications, Birkhäuser, Basel, Switzerland, 2013.

[6] V. Kokilashvili and M. Krbec, Weighted inequalities in Lorentz and Orlicz spaces, World Scientific, River Edge, NJ, USA, 1991.

[7] I. Genebashvili, A. Gogatishvili, V. Kokilashvili, and M. Krbec, Weight Theory for Integral Transforms on Spaces of Homogeneous Type, vol. 92 of Pitman Monographs and Surveys in Pure and Applied Mathematics, Longman, Harlow, UK, 1998.

[8] L. Diening, P. Harjulehto, P. Hästö, and M. Růžička, Lebesgue and Sobolev spaces with variable exponents, vol. 2017 of Lecture Notes in Mathematics, Springer, Berlin, Germany, 2011.

[9] A. Meskhi, Measure of Non-Compactness for Integral Operators in Weighted Lebesgue Spaces, Nova Science Publishers, New York, NY, USA, 2009.

[10] S. Samko, "On a progress in the theory of Lebesgue spaces with variable exponent: maximal and singular operators," Integral Transforms and Special Functions., vol. 16, no. 5-6, pp. 461-482, 2005.

[11] L. Pick and M. Růžička, "An example of a space $L^{p(x)}$ on which the Hardy-Littlewood maximal operator is not bounded," Expositiones Mathematicae, vol. 19, no. 4, pp. 369-371, 2001.

[12] D. Cruz-Uribe, L. Diening, and A. Fiorenza, "A new proof of the boundedness of maximal operators on variable Lebesgue spaces," Bollettino dell'Unione Matematica Italiana, Serie 9, vol. 2, no. 1, pp. 151-173, 2009.

[13] A. K. Lerner, "Some remarks on the Hardy-Littlewood maximal function on variable $L^{p}$ spaces," Mathematische Zeitschrift, vol. 251, no. 3, pp. 509-521, 2005.

[14] A. K. Lerner, "On some questions related to the maximal operator on variable $L^{p}$ spaces," Transactions of the American Mathematical Society, vol. 362, no. 8, pp. 4229-4242, 2010.

[15] E. Kapanadze and T. Kopaliani, "A note on maximal operator on $L^{p(t)}(\Omega)$ spaces," Georgian Mathematical Journal, vol. 15, no. 2, pp. 307-316, 2008.
[16] L. Diening, P. Harjulehto, P. Hästö, Y. Mizuta, and T. Shimomura, "Maximal functions in variable exponent spaces: limiting cases of the exponent," Annales Academice Scientiarum Fennicae Mathematica, vol. 34, no. 2, pp. 503-522, 2009.

[17] C. Fefferman and E. M. Stein, "Some maximal inequalities," American Journal of Mathematics, vol. 93, pp. 107-115, 1971.

[18] C. Pérez and R. L. Wheeden, "Uncertainty principle estimates for vector fields," Journal of Functional Analysis, vol. 181, no. 1, pp. 146-188, 2001.

[19] N. Trudinger, "On imbeddings into Orlicz spaces and some applications," Journal of Mathematics and Mechanics, vol. 17, pp. 473-483, 1967.

[20] H. Triebel, The Structure of Functions, Birkhäuser, Basel, Switzerland, 2001.

[21] D. E. Edmunds, H. Hudzik, and M. Krbec, "On weighted critical imbeddings of Sobolev spaces," Mathematische Zeitschrift, vol. 268, no. 1-2, pp. 585-592, 2011.

[22] A. Fiorenza, "Regularity results for minimizers of certain one-dimensional Lagrange problems of calculus of variations," Bollettino dell'Unione Matematica Italiana, Serie 7, vol. 10-B, no. 4, pp. 943-962, 1996.

[23] G. Anatriello and A. Fiorenza, "Fully measurable grand Lebesgue spaces," Journal of Mathematical Analysis and Applications, vol. 422, no. 2, pp. 783-797, 2015.

[24] D. Cruz-Uribe and A. Fiorenza, "The $A_{\infty}$ property for young functions and weighted norm inequalities," Houston Journal of Mathematics, vol. 28, no. 1, pp. 169-182, 2002.

[25] M. M. Rao and Z. D. Ren, Theory of Orlicz Spaces, Marcel Dekker, 1991.

[26] A. Fiorenza, M. Krbec, and H.-J. Schmeisser, "An improvement of dimension-free Sobolev imbeddings in r.i. spaces," Journal of Functional Analysis, vol. 267, no. 1, pp. 243-261, 2014.

[27] J. Martín and M. Milman, "Integral isoperimetric transference and dimensionless Sobolev inequalities," Revista Matemática Complutense, vol. 28, no. 2, pp. 359-392, 2015. 


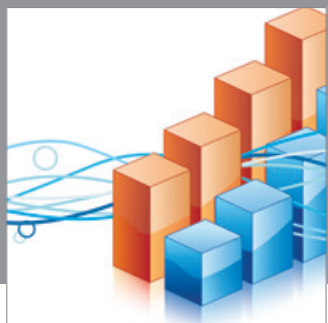

Advances in

Operations Research

mansans

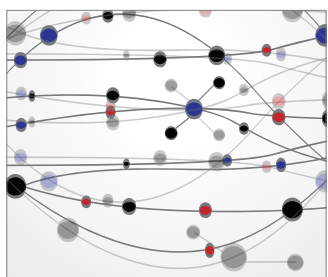

The Scientific World Journal
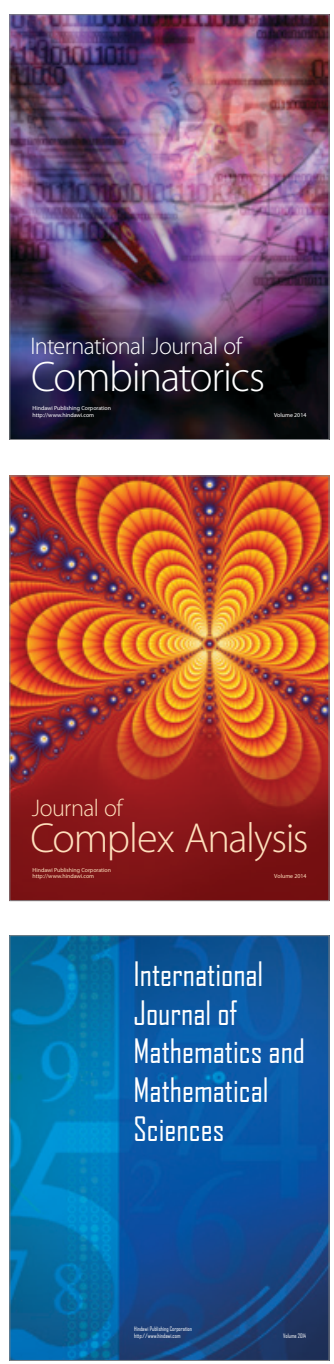


Submit your manuscripts at http://www.hindawi.com
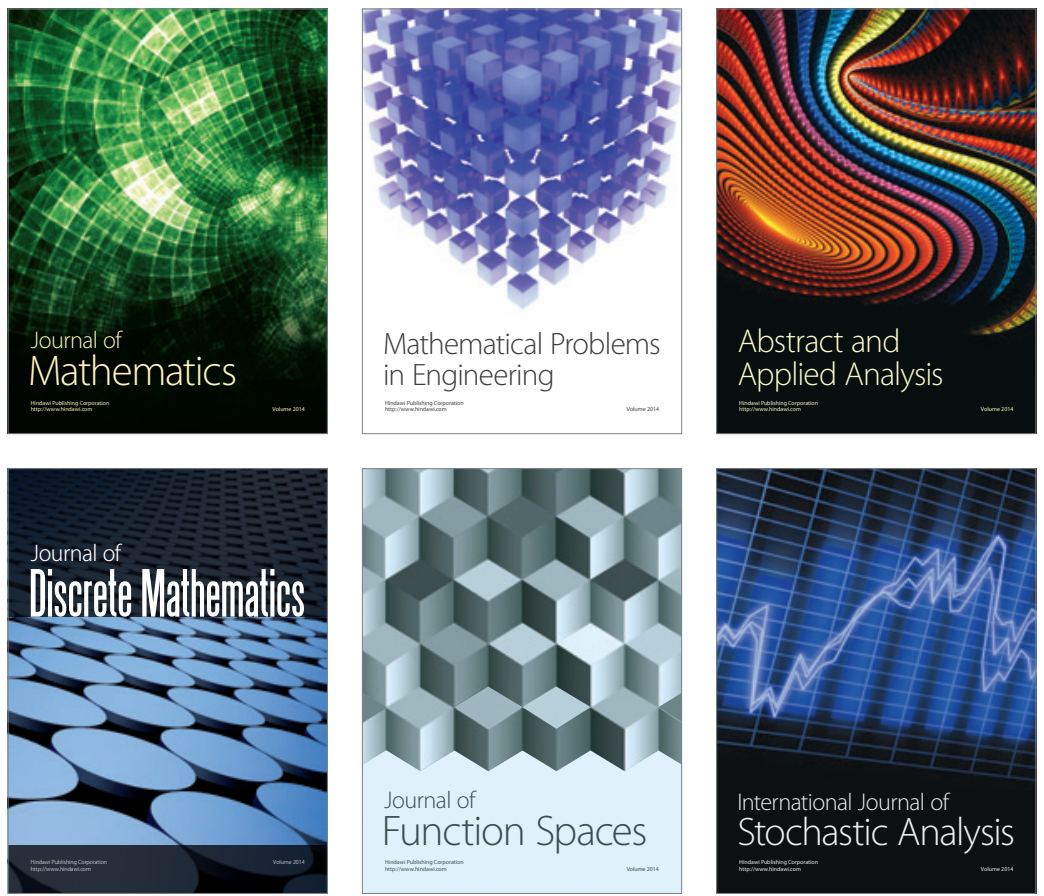

Journal of

Function Spaces

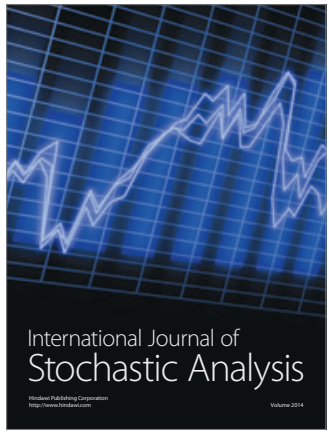


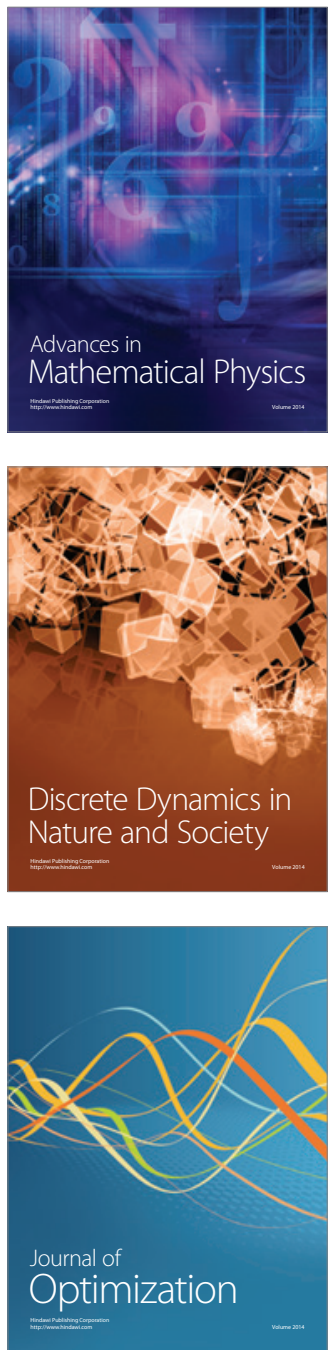